\title{
On infinitesimal projective transformations of a Riemannian manifold with constant scalar curvature
}

\author{
By Kazunari Yamauchi \\ (Received September 20, 1978; Revised February 13, 1979)
}

\section{$\S 1$. Introduction}

Let $M$ be a connected differentiable Riemannian manifold of dimension $n$ and $g_{j i}, \nabla_{j}, K_{k j i}{ }^{h}, K_{j i}$ and $K$, respectively, the components of the metric tensor, the operator of covariant differentiation with respect to the LeviCivita connection, the curvature tensor, the Ricci tensor and the scalar curvature, here and hereafter the indices $a, b, c, \cdots, i, j, k, \cdots$, run over the range $1,2,3, \cdots, n$. We shall denote $g^{j a} \nabla_{a}$ by $\nabla^{j}$. An infinitesimal transformation $v^{h}$ on $M$ is said to be projective if it satisfies,

$$
\mathcal{L}\left\{\begin{array}{c}
h \\
j
\end{array} i\right\}=\nabla_{j} \nabla_{i} v^{h}+K_{a j i}{ }^{h} v^{a}=\delta_{j}^{h} \varphi_{i}+\delta_{i}^{h} \varphi_{j},
$$

where $\mathcal{L}$ denotes the operator of Lie derivative with respect to $v^{h}\left\{\begin{array}{c}h \\ j\end{array}\right\}$ the Christoffel's symbol, $\delta_{j}^{h}$ the Kronecker's delta and $\varphi_{i}$ the associated gradient vector, respectively. In $(1-1)$, if we put $\nabla_{i} v^{i}=(n+1) f$, then we have $f_{i}=\varphi_{i}$, where $f_{i}$ means $\nabla_{i} f$, thus in the following discussions, we use $f_{i}$ instead of $\varphi_{i}$.

For the infinitesimal projective transformations, the following results are known.

TheOREM A. Let $M$ be a complete Riemannian manifold with parallel Ricci tensor. If $M$ admits nonaffin infinitesimal projective transformations, then $M$ is a space of positive constant curvature. [6], [9], [11].

TheOREM B. Let $M$ be a compact Riemannian manifold with constant scalar curvature. If the scalar curvature is nonpositive, then an infinitesimal projective transformation is a motion. [14].

The purpose of this paper is to prove the following theorem.

THEOREM. Let $M$ be a compact, connected and simply connected $n$ dimensional, $(n>2)$, Riemannian manifold with constant scalar curvature $K$. If $M$ admits nonisometric infinitesimal projective transformations, then 
$M$ is isometric to a sphere of radius $\sqrt{n(n-1) / K}$.

The following theorem is well known.

THEOREM C. Let $M$ be a complete, connected and simply connected Riemannian manifold of dimension $n$. In order that $M$ admits a nontrivial solution $\phi$ for the system of differential equations,

$$
\nabla_{k} \nabla_{j} \psi_{i}+k\left(2 \phi_{k} g_{j i}+\phi_{j} g_{i k}+\phi_{i} g_{k j}\right)=0, k>0 \text {, }
$$

where $\phi_{i}=\nabla_{i} \phi$, it is necessary and sufficient that $M$ be isometric with a sphere of radius $\sqrt{1 / k}$. [7], [10].

We have proved the following theorems.

THEOREM D. If the projective Killing vector $v^{h}$ can be decomposed as follows,

$$
v^{h}=w^{h}-\frac{n(n-1)}{2 K} f^{h}
$$

then $f^{h}$ satisfies the differential equation of (1-2), where $w^{h}$ is the Killing vector and the scalar curvature $K$ is positive constant [15].

THEOREM E. In a compact Riemannian manifold with positive constant scalar curvature, the projective Killing vector $v^{h}$ is decomposable as follows,

$$
v^{h}=w^{h}-\frac{n(n-1)}{2 K} f^{h}
$$

if and only if $G_{a i} f^{a}=0$, where $w^{h}$ is the Killing vector and $G_{j i}=K_{j i}$ $-\frac{K}{n} g_{j i}$ [15].

First of all we shall prove the following propositions.

Porposition 1. In a Riemannian manifold with constant scalar curvature, there is the following equation.

$$
2 G_{a i} f^{a}+(n-1) F_{i}=0,
$$

where $F=\Delta f+\frac{2(n+1)}{n(n-1)} K f, F_{i}$ means $\nabla_{i} F$ and $\Delta f$ denotes $\nabla_{i} f^{i}$.

PROPOSITION 2. In a compact Riemannian manifold with constant scalar curvature, we have the following equation,

$$
\int G_{a b} f^{a} f^{b} d \sigma=\frac{n-1}{2} \int F^{2} d \sigma,
$$

where do denotes the volume element. 
Thus from Proposition 2, Proposition 1, Theorem E, Theorem D and Theorem $\mathrm{C}$, to prove the Theorem, it is sufficient to show that $\int G_{a b} f^{a} f^{b} d \sigma$ is nonpositive.

From (1-1), we have the following equations,

$$
\begin{aligned}
& \mathcal{L} K_{k j i}{ }^{h}=-\delta_{k}^{h} \nabla_{j} f_{i}+\delta_{j}^{h} \nabla_{k} f_{i}, \\
& \mathcal{L} K_{j i}=-(n-1) \nabla_{j} f_{i}, \\
& \mathcal{L} K=-2 \nabla^{a} v^{b} K_{a b}-(n-1) \Delta f, \\
& \nabla^{a} \nabla_{a} v_{i}+K_{a i} v_{i}=2 f_{i}, \\
& \nabla^{a} \nabla_{i} v_{a}-K_{a i} v^{a}=(n+1) f_{i} .
\end{aligned}
$$

\section{$\S 2$. Proof of Proposition 1.}

Lemma 1. There exists the following equation,

$$
\mathcal{L} g^{a b} \nabla_{i} K_{a b}=0 .
$$

Proof. From (1-1) and (1-6), we have

$$
\begin{aligned}
& 0=\nabla^{j}\left(\nabla_{j} \nabla_{k} v_{i}+K_{a j k i} v^{a}-g_{j i} f_{k}-g_{i k} f_{j}\right) \\
& =\nabla^{j} \nabla_{j} \nabla_{k} v_{i}+\nabla_{j} K_{k i a}{ }^{j} v^{a}+K_{a j_{k} i} \nabla^{j} v^{a}-\nabla_{i} f_{k}-g_{i k} \Delta f \\
& =\nabla^{j}\left(\nabla_{k} \nabla_{j} v_{i}-K_{j k i}{ }^{a} v^{a}\right)+\left(\nabla_{k} K_{i a}-\nabla_{i} K_{k a}\right) v^{a}+K_{a j k i} \nabla^{j} v^{a} \\
& -\nabla_{i} f_{k}-g_{i k} \nabla f \\
& =\nabla^{j} \nabla_{k} \nabla_{j} v_{i}-\nabla_{j} K_{a i k}{ }^{j} v^{a}-K_{j k i a} \nabla^{j} v^{a}+\left(\nabla_{k} K_{i a}-\nabla_{i} K_{k a}\right) v^{a} \\
& +K_{a j_{k} i} \nabla^{j} v^{a}-\nabla_{i} f_{k}-g_{i k} \Delta f \\
& =\nabla_{k} \nabla^{j} \nabla_{j} v_{i}-K^{j}{ }_{k j}{ }^{a} \nabla_{a} v_{i}-K^{j}{ }_{k i}^{a} \nabla_{j} v_{a}-\left(\nabla_{a} K_{i k}-\nabla_{i} K_{a k}\right) v^{a} \\
& +\left(K_{a j k i}-K_{j k i a}\right) \nabla^{j} v^{a}+\left(\nabla_{k} K_{i a}-\nabla_{i} K_{k a}\right) v^{a}-\nabla_{i} f_{k} \\
& -g_{i k} \Delta f \\
& =\nabla_{k}\left(2 f_{i}-K_{i a} v^{a}\right)+K_{k}^{a} \nabla_{a} v_{i}+\left(K_{a j k i}-2 K_{j k i a}\right) \nabla^{j} v^{a} \\
& +\left(\nabla_{k} K_{i a}-\nabla_{a} K_{i k}\right) v^{a}-\nabla_{i} f_{k}-g_{i k} \Delta f \\
& =\nabla_{k} f_{i}-K_{i a} \nabla_{k} v^{a}+K_{k}^{a} \nabla_{a} v_{i}+\left(K_{a j k i}-2 K_{j k i a}\right) \nabla^{j} v^{a} \\
& -\nabla_{a} K_{i k} v^{a}-g_{i k} \Delta f \text {. }
\end{aligned}
$$

Operate $\nabla^{k}$ on the above equation, we obtain the following equation by means of (1-1), (1-6), $\nabla^{k} \nabla_{k} f_{i}=\nabla_{i}(\Delta f)+K_{i a} f^{a}$, and $\nabla^{k} K_{i k}=\frac{1}{2} \nabla_{i} K=0$, 


$$
\begin{aligned}
& 0=\nabla^{k} \nabla_{k} f_{i}-\nabla_{k} K_{i a} \nabla^{k} v^{a}-K_{i a} \nabla^{k} \nabla_{k} v^{a}+K_{k}^{a} \nabla^{k} \nabla_{a} v^{i} \\
& +\left(\nabla_{k} K_{j a i}{ }^{k}-2 \nabla_{k} K_{i a j}{ }^{k}\right) \nabla^{j} v^{a}+\left(K_{a j k i}-2 K_{j k i a}\right) \nabla^{k} \nabla^{j} v^{a} \\
& -\nabla^{k} \nabla_{a} K_{i k} v^{a}-\nabla_{a} K_{i k} \nabla^{k} v^{a}-\nabla_{i}(\Delta f) \\
& =\nabla_{i}(\Delta f)+K_{i a} f^{a}-\nabla_{k} K_{i a} \nabla^{k} v^{a}-K_{i a}\left(2 f^{a}-K_{b}^{a} v^{b}\right) \\
& +K_{k}^{a}\left(-K_{b}{ }_{a i} v^{b}+\delta_{i}^{k} f_{a}+g_{a i} f^{k}\right)+\left(\nabla_{j} K_{a i}+\nabla_{a} K_{i j}\right. \\
& \left.-2 \nabla_{i} K_{a j}\right) \nabla^{j} v^{a}+\left(K_{a j k i}-2 K_{j k i a}\right)\left(-K_{b}{ }^{k j a} v^{b}+g^{k a} f^{j}+g^{j a} f^{k}\right) \\
& -\left(\nabla_{a} \nabla^{k} K_{i k}-K_{a i}^{k} K_{b k}-K_{a k}^{k}{ }^{b} K_{i b}\right) v^{a}-\nabla_{a} K_{i k} \nabla^{k} v^{a}-\nabla_{i}(\Delta f) \\
& =\nabla_{i} K_{a b} \mathcal{L} g_{a b}-\left(K_{a j_{k} i}-2 K_{j_{k i a}}\right) K^{b k j a} v_{b} \text {. }
\end{aligned}
$$

On the orther hand, since $K_{j k i a}+K_{j i a_{k}}+K_{j a_{k} i}=0$, we have

$$
\begin{aligned}
\left(K_{a j k i}-2 K_{j k i a}\right) K^{b k j a} & =\left(K_{j i a k}-K_{j k i a}\right) K^{b k j a} \\
& =K_{j i a k} K^{b k j a}-K_{a i k j} K^{b k j a} \\
& =K_{j i a k} K^{b k j a}-K_{j i a k} K^{b a k j} \\
& =K_{j i a k} K^{b k j a}+K_{j i k a} K^{b a k j} \\
& =K_{j i a k} K^{b k j a}+K_{j i a k} K^{b k a j} \\
& =K_{j i a k}\left(K^{b k j a}+K^{b k a j}\right) \\
& =0 .
\end{aligned}
$$

Therefore Lemma 1 is proved.

Since the scalar curvature is constant and from Lemma 1, we have

$$
\begin{aligned}
& 0=\mathcal{L}\left(g^{a b} \nabla_{i} K_{a b}\right) \\
& =g^{a b} \mathcal{L} \nabla_{i} K_{a b}+\nabla_{i} K_{a b} \mathcal{L} g^{a b} \\
& =g^{a b} \mathcal{L} \nabla_{i} K_{a b}
\end{aligned}
$$

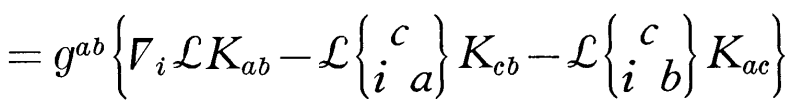

$$
\begin{aligned}
& =g^{a b}\left\{-(n-1) \nabla_{i} \nabla_{a} f_{b}-\left(\delta_{i}^{c} f_{a}+\delta_{a}^{c} f_{i}\right) K_{c b}-\left(\delta_{i}^{c} f_{b}+\delta_{b}^{c} f_{i}\right) K_{a c}\right\} \\
& =-2 G_{i a} f^{a}-(n-1) F_{i} \text {. }
\end{aligned}
$$

Thus Proposition 1 is proved.

\section{$\S 3 . \quad$ Proof of Proposition 2.}

LEMMA 2. We have the following equation,

$$
\nabla^{a} \nabla_{a} f_{i}=\frac{n-3}{n-1} G_{i a} f^{a}-\frac{n+3}{n(n-1)} K f_{i} .
$$

Proof. From Proposition 1, we obtain 


$$
\nabla_{i}(\Delta f)=-\frac{2}{n-1} G_{i a} f^{a}-\frac{2(n+1)}{n(n-1)} K f_{i} .
$$

Therefore we have

$$
\begin{aligned}
\nabla^{a} \nabla_{a} f_{i} & =\nabla^{a} \nabla_{i} f_{a} \\
& =\nabla_{i}(\Delta f)-K^{a}{ }_{i a}^{b} f_{b} \\
& =\frac{n-3}{n-1} G_{i a} f^{a}-\frac{n+3}{n(n-1)} K f_{i} .
\end{aligned}
$$

Thus Lemma 2 is proved.

Lemma 3. There exists the following equation,

$$
\int\left\{-\frac{n-2}{n-1} G_{j i} v^{i} f^{i}+\frac{2 K}{n(n-1)} f^{i} v_{i}+f^{i} f_{i}\right\} d \sigma=0 .
$$

Proof. From Lemma 2 and (1-6), we have

$$
\begin{aligned}
\nabla^{j} \nabla_{j}\left(f^{i} v_{i}\right)= & \nabla^{j}\left(\nabla_{j} f^{i} v_{i}+f^{i} \nabla_{j} v_{i}\right) \\
= & \nabla^{j} \nabla_{j} f^{i} v_{i}+2 \nabla_{j} f_{i} \nabla^{i} v^{i}+f^{i} \nabla^{j} \nabla_{j} v_{i} \\
= & \frac{n-3}{n-1} G_{j i} f^{j} v^{i}-\frac{n+3}{n(n-1)} K f^{i} v_{i}+2 \nabla_{j} f_{i} \nabla^{j} v^{i} \\
& \quad+f^{i}\left(2 f_{i}-K_{j i} v^{j}\right) \\
= & -\frac{2}{n-1} G_{j i} f^{j} v^{i}-\frac{2(n+1)}{n(n-1)} K f^{i} v_{i}+2 f^{i} f_{i} \\
& \quad+2\left\{\nabla^{j}\left(v^{i} \nabla_{j} f_{i}\right)-v^{i} \nabla^{j} \nabla_{j} f_{i}\right\} \\
=- & \frac{2(n-2)}{n-1} G_{j i} f^{j} v^{i}+\frac{4}{n(n-1)} K f^{i} v_{i}+2 f^{i} f_{i}+2 \nabla^{j}\left(v^{i} \nabla_{j} f_{i}\right) .
\end{aligned}
$$

Thus we have Lemma 3 by means of Green's Lemma.

Lemma 4. There is the following equation,

$$
\int\left\{\frac{2}{n-1} G_{j i} f^{j} v^{i}+\frac{2(n+1)}{n(n-1)} K f^{i} v_{i}+(n+1) f^{i} f_{i}\right\} d \sigma=0 .
$$

Proof. From Lemma 3 and (1-7), we have

$$
\begin{aligned}
\nabla_{j}\left(f^{i} \nabla_{j} v^{j}\right)= & \nabla_{j} f^{i} \nabla_{i} v^{j}+f^{i} \nabla_{j} \nabla_{i} v^{j} \\
= & \nabla_{i}\left(\nabla_{j} f^{i} v^{j}\right)-v^{j} \nabla_{i} \nabla_{j} f^{i}+f^{i}\left\{(n+1) f_{i}+K_{i j} v^{i}\right\} \\
= & \nabla_{i}\left(\nabla_{j} f^{i} v^{i}\right)-v^{i}\left\{\frac{n-3}{n-1} G_{j i} f^{i}-\frac{n+3}{n(n-1)} K f_{j}\right\} \\
& \quad+(n+1) f^{i} f_{i}+K_{j i} v^{j} f^{i}
\end{aligned}
$$




$$
\begin{aligned}
& =\nabla_{i}\left(\nabla_{j} f^{i} v^{j}\right)+\frac{2}{n-1} G_{j i} v^{j} f^{i}+\frac{2(n+1)}{n(n-1)} K f^{i} v_{i} \\
& \quad+(n+1) f^{i} f_{i} .
\end{aligned}
$$

Thus Lemma 4 is proved.

Lemma 5. We have the following equation,

$$
\int\left\{\frac{2}{n(n-1)} K f^{i} v_{i}+f^{i} f_{i}\right\} d \sigma=0 .
$$

Proof. From Lemma 3 and 4, proof is obvious.

LEMMA 6. There exists the following equation,

$$
\int\left\{f^{i} v_{i}+(n+1) f^{2}\right\} d \sigma=0 .
$$

PRoOF. $\nabla_{i}\left(f v^{i}\right)=f^{i} v_{i}+f \nabla_{i} v^{i}$

$$
=f^{i} v_{i}+(n+1) f^{2} .
$$

Thus Lemma 6 is proved.

LEMMA 7. There is the following equation,

$$
2 \int G_{j i} f^{j} f^{i} d \sigma=(n-1) \int \Delta f F d \sigma .
$$

Proof. From Proposition 1, we have

$$
\begin{aligned}
2 G_{j i} f^{j} f^{i} & =-(n-1) f_{i} F^{i} \\
& =-(n-1)\left\{\nabla_{i}\left(f^{i} F\right)-\Delta f F\right\} .
\end{aligned}
$$

Thus Lemma 7 is proved.

LEMMA 8. We have the following equation,

$$
\int f F d \sigma=0 .
$$

Proof. From Lemma 6, we obtain

$$
\int f_{i} v^{i} d \sigma=-(n+1) \int f^{2} d \sigma .
$$

Substituting this equation into the equation of Lemma 5, we have

$$
\begin{aligned}
0 & =\int\left\{\frac{2 K}{n(n-1)} f_{i} v^{i}+f_{i} f^{i}\right\} d \sigma \\
& =-\int\left\{\frac{2(n+1)}{n(n-1)} K f^{2}-\nabla_{i}\left(f f^{i}\right)+f \Delta f\right\} d \sigma \\
& =-\int f F d \sigma .
\end{aligned}
$$


Thus Lemma 8 is proved.

From Lemma 7 and Lemma 8, we have

$$
\begin{aligned}
\int F^{2} d \sigma & =\int F\left(\Delta f+\frac{2(n+1)}{n(n-1)} K f\right) d \sigma \\
& =\frac{2}{n-1} \int \mathrm{G}_{j i} f^{j} f^{i} d \sigma .
\end{aligned}
$$

Therefore Proposition 2 is proved.

\section{$\S 4$. Proof of Theorem.}

LEMMA 9. There is the following equation,

$$
\int \nabla_{j} f_{i} \nabla^{j} f^{i} d \sigma=\int\left\{-\frac{n-3}{n-1} G_{j i} f^{j} f^{i}+\frac{n+3}{n(n-1)} K f_{i} f^{i}\right\} d \sigma .
$$

Proof. From Lemma 2, we have

$$
\begin{aligned}
\nabla_{j} f_{i} \nabla^{j} f^{i} & =\nabla_{j}\left(f_{i} \nabla^{j} f^{i}\right)-f_{i} \nabla_{j} \nabla^{j} f^{i} \\
& =\nabla_{j}\left(f_{i} \nabla^{j} f^{i}\right)-\frac{n-3}{n-1} G_{j i} f^{j} f^{i}+\frac{n+3}{n(n-1)} K f_{i} f^{i} .
\end{aligned}
$$

Thus Lemma 9 is proved.

Lemma 10. There exists the following equation,

$$
-2 \int \nabla_{j} f_{i} \nabla^{j} v^{i} d \sigma=(n+3) \int f_{i} f^{i} d \bar{\sigma} .
$$

Proof. $\quad-2 \nabla_{j} f_{i} \nabla^{j} v^{i}=\nabla_{j} f_{i} \mathcal{L} g^{j i}$

$$
\begin{aligned}
& =\nabla_{j}\left(f_{i} \mathcal{L} g^{j i}\right)-f_{i} \nabla_{j} \mathcal{L} g^{j i} \\
& =\nabla_{j}\left(f_{i} \mathcal{L} g^{j i}\right)-f_{i}\left\{\mathcal{L} \nabla_{j} g^{j i}-\mathcal{L}\left\{\begin{array}{c}
j \\
j
\end{array}\right\} g^{a i}\right. \\
& \left.-\mathcal{L}\left\{\begin{array}{c}
i \\
j
\end{array}\right) g^{j a}\right\} \\
& =\nabla_{j}\left(f_{i} \mathcal{L} g^{j i}\right)+(n+3) f_{i} f^{i} \text {. }
\end{aligned}
$$

Thus Lemma 10 is proved.

LEMMA 11. We have the following equation,

$$
\int\left(\nabla_{j} v_{i} \nabla^{j} v^{i}+\nabla_{j} v_{i} \nabla^{i} v^{j}\right) d \sigma=-(n+3) \int f_{i} v^{i} d \sigma
$$

PRoof. $\nabla_{j} v_{i} \nabla^{j} v^{i}+\nabla_{j} v_{i} \nabla^{i} v^{j}$

$$
=-\nabla_{j} v_{i} \mathcal{L} g_{j i}
$$




$$
\begin{aligned}
= & -\nabla_{j}\left\{v_{i} \mathcal{L} g^{j i}\right\}+v_{i} \nabla_{j} \mathcal{L} g^{j i} \\
& =-\nabla^{j}\left(v_{i} \mathcal{L} g^{j i}\right)+v_{i}\left\{\mathcal{L} \nabla_{j} g^{j i}-\mathcal{L}\left\{\begin{array}{c}
j \\
j
\end{array}\right)\right\} g^{a i} \\
& \left.-\mathcal{L}\left\{\begin{array}{c}
i \\
j
\end{array}\right) g^{j a}\right\} \\
& =-\nabla_{j}\left(v_{i} \mathcal{L} g^{j i}\right)-(n+3) f_{i} v^{i} .
\end{aligned}
$$

Thus Lemma 11 is proved.

If we put

$$
P_{j i}=\nabla_{j} f_{i}+\frac{K}{n(n-1)}\left(\nabla_{j} v_{i}+\nabla_{i} v_{j}\right)
$$

then we have

$$
\begin{aligned}
& P_{j i} P^{j i}=\nabla_{j} f_{i} \nabla^{j} f^{i}+\frac{4 K}{n(n-1)} \nabla_{j} f_{i} \nabla^{j} v^{i}+\frac{2 K^{2}}{n^{2}(n-1)^{2}}\left(\nabla_{j} v_{i} \nabla^{j} v^{i}\right. \\
& \left.+\nabla_{j} v_{i} \nabla^{i} v^{j}\right) .
\end{aligned}
$$

Thus by means of Lemma 9, 10, 11, and 5, we have

$$
\int P_{j i} P^{j i} d \sigma=-\frac{n-3}{n-1} \int G_{j i} f^{j} f^{i} d \sigma
$$

Therefore if $\operatorname{dim} M=n \geqq 4$, then $\int G_{j i} f^{j} f^{i} d \sigma$ is nonpositive, and Theorem is proved. If $\operatorname{dim} M=n=3$, then we have

$$
P_{j i}=\nabla_{j} f_{i}+\frac{K}{n(n-1)}\left(\nabla_{j} v_{i}+\nabla_{j} v_{i}\right)=0
$$

Thus we have

$$
\begin{aligned}
0 & =g^{j i} P_{j i} \\
& =\Delta f+\frac{2(n+1)}{n(n-1)} K f \\
& =F .
\end{aligned}
$$

In this case, from Proposition 1, Theorem E, Theorem D and Theorem C, Theorem is proved. Therefore Theorem is completely proved.

\section{Bibliography}

[1] Akbar-Zadeh, Hassan and R. Couty: Espaces à tensor de Ricci parallele admettant des transformations projectives, C. R. Acad. Paris Sér, A-B 284 (1977), No. 15, A 891-A 893. 
[2] R. Couty: Transformations projective sur un espace d'Einstein complet, C. R. Acad. Sci. Paris Sér. A-B 252, 109-1097 (1961).

[3] R. Couty: Formes projectives fermées sur un espace d'Einstein, C. R. Acad. Sci. Paris Sér. A-B270, A 1249-1251 (1970).

[4] S. IsHIHARA: Groups of projectiv transformations on a projectively connected manifold, Japan J. Math. 25, 37-80 (1955).

[5] S. IsHIHARA: Groups of projective tronsformations and groups of conformal transformations, J. Math. Soc. Japan. 9, 195-227 (1957).

[6] T. NAGANO: The projective transformation on a space with parallel Ricci tensor, Kodai Math. Sem. Rep. 11, 131-138 (1959).

[7] M. Oвata: Riemannian manifolds admitting a solutionaof certain system of differential equations, Proc. U. S.-Japan Sem. in Differential Geom. Kyoto, Japan, (1965), 101-114.

[8] A. S. Solodovnikov: The group of projective transformations in a complete analytic Riemannian space, Dokl. Akad. Nauk. SSSR, 186, 1262-1265 (1969).

[9] N. TANAKA: Projective connections and projective transformations, Nagoya Math. J. 11, 1-24 (1957).

[10] S. TANNo: Differential equations of order 3 on Riemannian manifolds, (technical report).

[11] Y. TASHIRO: On projective transformations of Riemmannian manifold, J. Math. Soc. Japan 11, 196-204 (1959).

[12] K. YANO: The theory of Lie derivative and its applications, Amsterdam NorthHolland (1957).

[13] K. YANO- T. NAGANO: Some theorems on projective and conformal transformations, Indag Math. 19, 451-458 (1957).

[14] K. Yamauchi: On infinitesimal projective transformations Hokkaido Math. J. Vol. 3. (1974), No. 2, 262-270.

[15] K. YAMAUCHI: On infinitesimal projective transformations satisfying the certain conditions, Hokkaido Math. J. Vol. 7 (1978) No. 1, 74-77.

Mathematical Institute

College of Liberal Arts

Kagoshima University 